\title{
Nonlinear Balance on an Equatorial Beta Plane
}

\author{
David J. Raymond \\ Physics Department and Geophysical Research Center \\ New Mexico Tech \\ Socorro, NM 87801
}

April 26, 2009

\section{Summary}

Extension of the nonlinear balance model to include the equator is examined here from a theoretical perspective. It is found that this model is ill-posed, in that zonally symmetric equatorial Kelvin waves of arbitrary amplitude can occur. Two possible solutions are outlined. The zonally averaged zonal momentum equation provides auxiliary information that can be imposed on the flow. This equation can be solved simultaneously with the other parts of the problem. Alternatively, when small, isolated disturbances can be assumed to have negligible effect on the mean flow, one can simply assume that zonal means remain unchanged.

\section{Introduction}

Since the Coriolis parameter goes to zero at the equator, it is usually supposed that balanced theories such as quasigeostrophy (Charney and Stern, 1962) and semigeostrophy (Hoskins, 1975) are of little or no use there. However, the nonlinear balance model (Lorenz, 1960; McWilliams, 1985; Raymond, 1992, hereafter R) is valid for all Rossby number, subject to certain other 
conditions being satisfied. Nonlinear balance should therefore be a useful approximation for certain phenomena at the equator.

$\mathrm{R}$ computed solutions to the nonlinear balance equation for the case in which the Coriolis force is identically zero, i. e., for infinite Rossby number, as well as for constant, non-zero Coriolis parameter. One would therefore suppose that extension of the theory to an equatorial beta plane would be trivial. Unfortunately this appears not to be true. Further evidence for this comes from Gent and McWilliams (1983a), who show that nonlinear balance models satisfactorily reproduce all the equatorial modes of Matsuno (1966) except Kelvin and gravity waves, but also state that these models are singular for the zonally symmetric mode. The purpose of this paper is to propose a method of dealing with this problem.

\section{Extended model}

$\mathrm{R}$ developed a potential vorticity form of the nonlinear balance equations in an east-west channel on an $f$ plane. The modifications required to extend $\mathrm{R}$ 's model to an equatorial beta plane are limited to the potential vorticity definition,

$$
\rho_{0} q=f \Gamma+f \frac{\partial \theta^{\prime}}{\partial z}+\Gamma \nabla_{h}^{2} \psi+X
$$

which now has a variable Coriolis parameter, $f=\beta y$, where $\beta$ is assumed constant, and the balance equation,

$$
\nabla_{h}^{2} \Sigma+\beta \frac{\partial \psi}{\partial y}+Z=0
$$

which has the additional term proportional to $\beta^{1}$.

In these equations

$$
X=\boldsymbol{\zeta} \cdot \nabla \theta^{\prime}=\nabla \cdot\left(\boldsymbol{\zeta} \theta^{\prime}\right),
$$

where $\boldsymbol{\zeta}$ is the approximate relative vorticity vector computed solely from the solenoidal part of the flow, and

$$
Z=2\left[\left(\frac{\partial^{2} \psi}{\partial x \partial y}\right)^{2}-\frac{\partial^{2} \psi}{\partial x^{2}} \frac{\partial^{2} \psi}{\partial y^{2}}\right]
$$

\footnotetext{
${ }^{1} \mathrm{R}$ included the divergence of an applied force on the right side of (2), but this term is often ignored. Inclusion in the present analysis would be straightforward.
} 
(See table 1 for notation.)

Note that this model is closer to the BE model of Gent and McWilliams (1983b) with $f$ set to $\beta y$ than to their $\beta \mathrm{BE}$ model, the difference being that $f$ is not set to a constant reference value where it is not differentiated.

As mentioned in the introduction, the problem lies in the zonally symmetric mode. Let us therefore isolate this mode by taking an $x$ or zonal average, indicated here by an overbar. A deviation from this average is indicated by a superscripted asterisk. Averaging (1) yields

$$
\rho_{0} \bar{q}=f \Gamma+f \frac{\partial \overline{\theta^{\prime}}}{\partial z}+\Gamma \frac{\partial^{2} \bar{\psi}}{\partial y^{2}}+\bar{X}
$$

where

$$
\bar{X}=\nabla \cdot\left(\bar{\zeta} \overline{\theta^{\prime}}+\overline{\zeta^{*} \theta^{*}}\right) .
$$

Since zonally averaging the $x$ derivative of anything yields zero because of the assumed periodic boundary conditions in $x$,

$$
\bar{Z}=\frac{\partial^{2}}{\partial y^{2}} \overline{\left(\frac{\partial \psi}{\partial x}\right)^{2}} \equiv \frac{\partial^{2} \bar{Q}}{\partial y^{2}} .
$$

Zonally averaging (2) and integrating in $y$ thus yields

$$
\theta_{0} \frac{\partial \bar{\sigma}}{\partial y}-f \frac{\partial \bar{\psi}}{\partial y}=0
$$

where $\bar{\sigma}=\bar{\pi}^{\prime}+\bar{Q} / \theta_{0}$, and where the constant of integration has been eliminated by assuming that geostrophic balance holds on the north and south boundaries of the domain, taken to be rigid walls.

Subtracting zonal means from (1) and (2) yields equations for perturbations from the mean:

$$
\begin{gathered}
\rho_{0} q^{*}=f \frac{\partial \theta^{*}}{\partial z}+\Gamma \nabla_{h}{ }^{2} \psi^{*}+X^{*}, \\
\nabla_{h}{ }^{2} \Sigma^{*}+\beta \frac{\partial \psi^{*}}{\partial y}+Z^{*}=0 .
\end{gathered}
$$

Equations (9) and (10) can be inverted to obtain perturbation fields, assuming that the nonlinear terms, $X^{*}$ and $Z^{*}$, which contain references to the zonally averaged fields, can somehow be evaluated. It is via these terms that zonal means affect the perturbation flow. The evolution of the zonal means may in principle be derived from jointly solving (5) and (8). 


\begin{tabular}{|l|l|}
\hline Symbol & Meaning \\
\hline$x, y, z, t$ & space and time variables \\
$\boldsymbol{k}$ & unit vector in $z$ direction \\
$\boldsymbol{\nabla}$ & gradient \\
$\boldsymbol{\nabla}_{h}$ & horizontal gradient \\
$g$ & acceleration of gravity \\
$f(y)$ & Coriolis parameter \\
$\beta$ & $d f / d y$ (constant) \\
$\xi$ & $\beta y^{2} / 2$ \\
$\rho_{0}(z)$ & ambient density profile \\
$\theta_{0}(z)$ & ambient potential temperature profile \\
$\Gamma(z)$ & $d \theta_{0} / d z$ \\
$N^{2}$ & $g \Gamma / \theta_{0}$ \\
$q$ & potential vorticity \\
$\theta^{\prime}$ & potential temperature perturbation \\
$\psi$ & horizontal streamfunction \\
$\pi^{\prime}$ & Exner function perturbation \\
$\Sigma$ & $\theta_{0} \pi^{\prime}-f \psi$ \\
$\boldsymbol{u}_{\psi}$ & solenoidal part of horizontal velocity field \\
$\boldsymbol{u}_{\chi}$ & irrotational part of horizontal velocity field \\
$u, v, w$ & $x, y$, and $z$ velocity components \\
$\boldsymbol{\zeta}$ & relative vorticity \\
$\bar{Q}$ & see equation $(7)$ \\
$X$ & see equation $(3)$ \\
$Z$ & see equation $(4)$ \\
$\bar{\sigma}$ & $\overline{\pi^{\prime}}+\bar{Q} / \theta_{0}$ \\
$\boldsymbol{F}$ & external body force \\
$\gamma$ & see equation (14) \\
$m$ & vertical wavenumber \\
\hline
\end{tabular}

Table 1: Definitions of symbols. 


\section{Zonally symmetric problem}

Equations (5) and (8) are nonlinear and inhomogeneous in barred quantities. It is instructive to examine solutions to the linearized, homogeneous versions of these equations. Insight into this simplified problem will carry over to the full problem.

Dropping $\bar{X}$ and $\bar{Q}$, setting $\bar{q}$ to its planetary value of $f \Gamma / \rho_{0}$, and combining (5), (8), and the hydrostatic equation, $\theta_{0} \partial \pi^{\prime} / \partial z=g \theta^{\prime} / \theta_{0}$, one finds that

$$
\frac{\Gamma}{f} \frac{\partial}{\partial y}\left(\frac{\theta_{0}}{f} \frac{\partial \overline{\pi^{\prime}}}{\partial y}\right)+\frac{\partial}{\partial z}\left(\frac{\theta_{0}^{2}}{g} \frac{\partial \overline{\pi^{\prime}}}{\partial z}\right)=0 .
$$

If $f, \theta_{0}$, and $\Gamma \equiv d \theta_{0} / d z$ are all approximately constant, then $\overline{\pi^{\prime}}$, after scaling, obeys Laplace's equation in two dimensions, which means that the maximum value of $\left|\overline{\pi^{\prime}}\right|$ occurs on the boundary of the solution domain, and a unique solution (up to an additive constant) can be obtained by specifying the normal derivative of $\overline{\pi^{\prime}}$ on the boundary. In the limit as $f \rightarrow 0$, (11) reduces to $\partial^{2} \overline{\pi^{\prime}} / \partial y^{2}=0$, and the above conclusion still holds.

On an equatorial beta plane, (11) can be approximately written

$$
N^{2} \frac{\partial^{2} \overline{\pi^{\prime}}}{\partial \xi^{2}}+\frac{\partial^{2} \overline{\pi^{\prime}}}{\partial z^{2}}=0,
$$

where $\xi=\beta y^{2} / 2$, and where $N=\left(g \Gamma / \theta_{0}\right)^{1 / 2}$ is the Brunt frequency. This is also in the form of Laplace's equation, but with a different north-south variable, $\xi$. If the equator is included in the computational domain, then $\overline{\pi^{\prime}}$ is double-valued, since a single value of $\xi$ represents two points, one north and one south of the equator. The physical problem therefore breaks down into two mathematical problems, namely solving Laplace's equation in two domains, one north and one south of the equator. Two conditions therefore need to be applied to $\overline{\pi^{\prime}}$ at the equator. Physically sensible choices are equality of $\overline{\pi^{\prime}}$ and $\partial \overline{\pi^{\prime}} / \partial \xi$ at the interface between the two domains. Since $d \xi=f d y$, (8) shows that the second condition is equivalent to continuity in the zonal wind across the equator. The first condition simply expresses continuity of pressure there.

Since the equator is on the boundary of the mathematical domain (i. e., at $\xi=0$ ), a minimum or maximum in $\overline{\pi^{\prime}}$ can reside there, even though the equator is actually inside the physical domain. Solutions may therefore 
exist in which the pressure perturbation is everywhere zero on the external physical boundaries (i. e., the north and south walls of the channel) but is non-zero in the (physical) interior, a situation that is impossible on an $f$ plane. As an example, solutions of (12) (with $N$ constant) of the form

$$
\overline{\pi^{\prime}}=m \exp (-m \xi / N+i m z)=m \exp \left[-m \beta y^{2} /(2 N)+i m z\right]
$$

demonstrate this possibility, and represent zonally symmetric Kelvin waves. By making the vertical wavenumber $m$ arbitrarily large, $\overline{\pi^{\prime}}$ can be made arbitrarily large on the equator, but arbitrarily small on the north and south boundaries. The boundary conditions on the north and south walls thus exert negligible control over the interior solution, and the mathematical problem is ill-posed.

\section{Proposed solutions}

One possible way to resolve the problem of uncontrolled zonally symmetric Kelvin waves is to impose on the solution an independent estimate of the zonally averaged zonal wind at the equator. This information may be obtained from the zonally averaged zonal momentum equation, which can be solved simultaneously with the other parts of the calculation. Since $\bar{u}=-\partial \bar{\psi} / \partial y$, this then provides a condition on $\partial \bar{\psi} / \partial y$, and hence on $\partial \overline{\pi^{\prime}} / \partial \xi$, at the equator.

Unfortunately, the approximations made in nonlinear balance models keep them from being precisely consistent with the standard momentum equation of fluid dynamics. However, Gent and McWilliams (1983b) showed that the Lorenz (1960) model is consistent with a modified horizontal momentum equation, which may be written

$$
\frac{d \boldsymbol{u}_{\psi}}{d t}+\boldsymbol{u}_{\psi} \cdot \nabla \boldsymbol{u}_{\chi}+\theta_{0} \nabla \pi^{\prime}+f \boldsymbol{k} \times \boldsymbol{u}=\boldsymbol{F}+\nabla \gamma
$$

in the notation of $\mathrm{R}$, with $\boldsymbol{u}_{\boldsymbol{\psi}}$ and $\boldsymbol{u}_{\boldsymbol{\chi}}$ being the solenoidal and irrotational parts of the horizontal wind. The scalar function $\gamma$ obeys a complex diagnostic equation in terms of the velocity components, which comes from demanding that the divergence of the above equation be consistent with the usual nonlinear balance condition. Fortunately, taking the $x$ average of the $x$ component of this equation eliminates $\gamma$, so this diagnostic equation does 
not have to be solved here. The result of the averaging process yields

$$
-\frac{\partial^{2} \bar{\psi}}{\partial t \partial y}-\frac{\partial}{\partial y}\left(\overline{v \frac{\partial \psi}{\partial y}}+\overline{\frac{\partial \psi}{\partial x} \frac{\partial \phi}{\partial x}}\right)-\frac{1}{\rho_{0}} \frac{\partial}{\partial z}\left(\rho_{0} \overline{w \frac{\partial \psi}{\partial y}}\right)-f \bar{v}=\bar{F}_{x} .
$$

This prognostic equation can be solved simultaneously with the rest of the calculation.

As $\mathrm{R}$ noted, the Lorenz model differs from the semibalanced model of $\mathrm{R}$ in that the vertical component of the absolute vorticity is used rather than the component normal to isentropic surfaces. Thus, (15) is slightly in error for the semibalanced model. However, the error should be considerably less than that resulting from the use of the exact $x$ momentum equation. Unfortunately, no equation equivalent to (14) for the semibalanced model is known to the author.

An alternative simpler than solving the zonally averaged zonal momentum equation is available when the problem consists of computing the approximate behavior of a small disturbance to an otherwise unperturbed large-scale zonal flow. In this case zonal averages of products of perturbed quantities, e. g., $\overline{\boldsymbol{\zeta}^{*} \theta^{*}}$, are small and can be ignored, resulting in

$$
\bar{X} \approx \nabla \cdot\left(\overline{\boldsymbol{\zeta}} \overline{\theta^{\prime}}\right)=\bar{\zeta}_{y} \frac{\partial \overline{\theta^{\prime}}}{\partial y}+\bar{\zeta}_{z} \frac{\partial \overline{\theta^{\prime}}}{\partial z}
$$

and

$$
\bar{Z} \approx \frac{\partial^{2}}{\partial y^{2}}\left(\frac{\partial \bar{\psi}}{\partial x}\right)^{2}=0
$$

In this approximation zonal means remain unchanged with time and the evolution of the system is confined to the perturbation fields.

\section{Discussion}

The results of this paper should be applicable not only to the nonlinear balance model on a beta plane, but to any three dimensional balanced model that includes the equator in the interior of its domain, since nothing peculiar to nonlinear balance is essential to the present analysis.

An alternative way to understand the difficulty in inverting a balanced model across the equator is to realize that Kelvin waves produce no perturbation in the potential vorticity field on isentropic surfaces. Thus, it is 
impossible to recover Kelvin waves in a potential vorticity inversion unless they are implicit in the boundary conditions at the equator. Since finite wavelength Kelvin modes are excluded from this model, only the zonally symmetric Kelvin mode can appear. It is interesting that while balanced models cannot directly predict the amplitude of this mode, the addition of just the principle of the conservation of angular momentum (or its balanced model equivalent) is sufficient to rectify this situation.

Though this paper demonstrates how to extend balanced models across the equator, it makes no statement as to the accuracy of such models relative to the primitive equations. Since finite wavelength Kelvin waves are definitely excluded from the model, its applicability will rest in part as to how important these waves are in the desired solution.

Programs to extend balanced models to the globe (e. g., Shutts, 1989) should benefit from this analysis.

Acknowledgments. Hongli Jiang's unexpected problems in extending our nonlinear balance model to equatorial regions pointed the way to this analysis. Bill Stone of the New Mexico Tech mathematics department helped clarify the nature of the difficulty at the equator while Wayne Schubert and Michael McIntyre both pointed out that Kelvin waves do not appear on IPV charts. The anonymous referees provided useful comments. This work was supported by National Science Foundation Grant No. ATM-8914116.

\section{References}

Charney, J. G., and M. E. Stern, 1962: On the stability of internal baroclinic jets in a rotating atmosphere. J. Atmos. Sci., 19, 159-172 (Corrigendum in McIntyre, 1972).

Gent, P. R., and J. C. McWilliams, 1983a: The equatorial waves of balanced models. J. Phys. Ocean., 13, 1179-1191.

Gent, P. R., and J. C. McWilliams, 1983b: Consistent balanced models in bounded and periodic domains. Dyn. Atmos. Oceans, 7, 67-93.

Hoskins, B. J., 1975: The geostrophic momentum approximation and the semi-geostrophic equations. J. Atmos. Sci., 32, 233-243.

Lorenz, E., N., 1960: Energy and numerical weather prediction. Tellus, 12, 364-373. 
Matsuno, T., 1966: Quasi-geostrophic motions in the equatorial area. $J$. Meteor. Soc. Japan, 44, 25-43.

McIntyre, M. E., 1972: Baroclinic instability of an idealized model of the polar night jet. Quart. J. Roy. Meteor. Soc., 98, 165-174.

McWilliams, J. C., 1985: A uniformly valid model spanning the regimes of geostrophic and isotropic, stratified turbulence: Balanced turbulence. J. Atmos. Sci., 42, 1773-1774.

Raymond, D. J., 1992: Nonlinear balance and potential vorticity thinking at large Rossby number. Quart. J. Roy. Meteor. Soc., 118, 987-1015.

Shutts, G. J., 1989: Planetary semi-geostrophic equations derived from Hamilton's principle. J. Fluid Mech., 208, 545-573. 